\title{
Agency attribution and visuospatial perspective taking
}

\author{
JAN ZWICKEL \\ Ludwig-Maximilians-Universität München, Munich, Germany
}

\begin{abstract}
We tested whether processes that evoke agency interpretations and mental state attributions also lead to adoption of the actor's visuospatial perspective by the observer. Agency and mental state interpretations were manipulated by showing different film clips involving two triangles (the Frith-Happé animations). Participants made speeded spatial decisions while watching these films. The responses in the spatial task could be either the same or different when given from the perspective of the participant versus the perspective of one of the triangles. Reaction times were longer when the perspectives of the participants and triangles differed than when they were the same. This effect increased as the need to invoke agency interpretations in order to understand the films increased, and it increased for those films that had previously been shown to evoke mental state attributions. This demonstrates that processing of an agent's behavior co-occurs with perspective adoption, even in the case in which triangles are the actors.
\end{abstract}

A crucial part of human life involves social interactions. To react adequately in these situations, it is important to take the representation of the world held by an interacting partner into account - for example, to understand what further information would be needed in a conversation or to predict actions on the basis of the assumed state of the other.

Therefore, it is not surprising to find that people are generally willing to represent the situations of others (Frith \& Frith, 2006) and to do so even if this involves representing painful stimulations (Jackson, Meltzoff, \& Decety, 2005). The ability to correctly represent what someone else knows requires that the visuospatial perspective (VSP) of the other be taken into account in order to understand what the other can or cannot know (Aichhorn, Perner, Kronbichler, Staffen, \& Ladurner, 2006). This can then be used as a starting state for predicting how the other person feels or will act (Apperly, 2008).

That VSP taking occurs spontaneously (independently of task requirements) in the presence of humans has been shown by Tversky and Hard (2009). Tversky and colleagues asked participants to describe the spatial relationship of two objects in a picture ("in relation to the bottle, where is the book?"). In one experimental condition, a human was seated behind the two objects and faced the observer. Therefore, the book was to the right of the bottle from the observer's perspective but to the left of the bottle when seen from the perspective of the depicted person. One picture was taken while the male actor was reaching for the book, and another picture when the actor was looking at the book but not reaching. The final picture showed the same situation without a human. When the pictures contained a human, observers often spontane- ously described the location of the book from the point of view of the depicted person. This tendency was further increased when the word "placed" was added to the question ("in relation to the bottle, where is the book placed?"), which, according to the authors, drew attention to the action and thereby increased the effect. These results were interpreted as showing that the participants spontaneously took the perspective of the depicted person to make sense of the situation.

Another demonstration of VSP taking in the presence of humans can be found in the study of Thomas, Press, and Haggard (2006). In the experiment, participants faced either a human model or an object (a house). The participants' task was to report a tactile cue that could be in either an anatomically same or a different position with respect to a visual cue presented on the human model or object. For example, a tactile cue to the participant's right arm could follow a visual cue on the model's right arm (anatomically the same) or on the model's left arm (same side, as seen from the participants' perspective). In the human model condition, the participants were faster for anatomically same than for different tactile-visual conditions, demonstrating that the perspective of the model played a role when the visual stimuli were coded. No difference between same and different situations was found in the object condition. One important difference between this and the present study is that only the present study involved movements. We expected that objects that display movement patterns that lead to the attribution of agency (Johnson, 2003) would also lead to VSP taking.

These reported studies show that VSP taking occurs spontaneously in the presence of a human, but it is unclear

J. Zwickel, zwickel@psy.uni-muenchen.de 
whether this is caused by the presence of a human body, as Thomas et al. (2006) suggested, or because the presence of a human is taken as a cue for the presence of an agent, which then causes VSP taking. The increase in VSP taking when attention was drawn to the action in Tversky and Hard (2009) suggests the latter, because human actions can be interpreted as cues of agency.

The present study should shed light on this question. The occurrence of VSP taking even if only nonhuman entities were present, as long as these entities seemed to be agents, would support the interpretation that VSP taking in the studies above occurred not because of the presence of a human but because the picture of the human acted as a cue to the presence of an agent. According to this reasoning, detecting an agent co-occurs with VSP taking.

In Experiment 1, we tested whether processes that invoked the interpretation of agency would also lead to VSP taking when no visual features of humans were present. To this end, we showed short film clips to the participants. These films were taken from the Frith-Happé animations and depicted a red and blue triangle moving in a self-propelled fashion across the screen (Abell, Happé, \& Frith, 2000). It has been shown before that these films activate agency and theory-of-mind (ToM; see below) processes to a different degree (e.g., Abell et al., 2000; Castelli, Happé, Frith, \& Frith, 2000; Klein, Zwickel, Prinz, \& Frith, 2009). Each film belongs to one of three categories: The films "Billiard," "Drifting," and "Tennis" belong to the random (R) category. They contain no interaction between the triangles. For example, in "Tennis," the triangles bounce back and forth in a rather uncoordinated way. According to typical descriptions of participants, the triangles float around without purpose.

The triangles in the films in the goal-directed (GD) category respond to one another's physical movements - for example, following each other continuously (chasing) or with stops in-between (leading), or moving around each other in a symmetric way (dancing). The movement of the triangles in these films can be described as fulfilling a certain goal ("the triangles danced around each other"), but it is not necessary to attribute a specific mental state to the triangles ("one triangle wanted to dance with the other").

Importantly, only the films in the ToM category typically lead to descriptions according to which the triangles react to each other's underlying mental states. One description of the ToM film "Mocking" may be that the small triangle is mocking the big triangle behind its back, but when the big triangle turns and, therefore, can see the small one, the small one pretends to do something else. A description of "Coaxing" may be that the big triangle is pushing the small triangle, which wants to stay inside, outside the house. Finally, the big triangle manages to move the small one outside. Snapshots of the film "Surprising" are depicted in Figure 1. Understanding of ToM animations therefore requires some attribution of mental states to the triangles.

VSP taking was expected to occur for films in the GD category, because the two triangles would be perceived as agents. If the attribution of mental states further increased the tendency toward VSP taking, this should result in larger effects for films in the ToM category than for those in the GD category. VSP taking was measured by asking participants to respond to dots occurring right or left of the red triangle with right and left keypresses, respectively. In the following, right and left will always refer to the observer's perspective, which was also how the participants were instructed to respond. These dots occurred while the red triangle's tip was pointing either upward or downward. If it was pointing upward, the triangle could be said to have a spatial orientation that matched the orientation of the participant. In this case, right and left decisions would be the same, whether or not participants adopt and respond relative to the spatial orientation of the triangle or their own. However, when the triangle was facing down, it might create interference, because if the perspective of the triangle were taken, the response would be incongruent to the response from the participant's perspective (see Figure 2). If agency/mental-state attribution co-occurred with a stronger tendency toward VSP taking, responses during downward-pointing directions in GD/ToM films should lead to more interference. This interference, in turn, should be reflected in longer reaction times (RTs).

\section{EXPERIMENT 1}

\section{Method \\ Participants, Apparatus, Stimuli, and Design. Twenty-four participants with normal or corrected-to-normal vision (13 females, mean age $=25$ years) were paid for participation and were naive with respect to the purpose of the study. An eyetracker was used to ensure that the participants fixated the middle of the screen at the beginning of each movie. Responses were collected with the left}

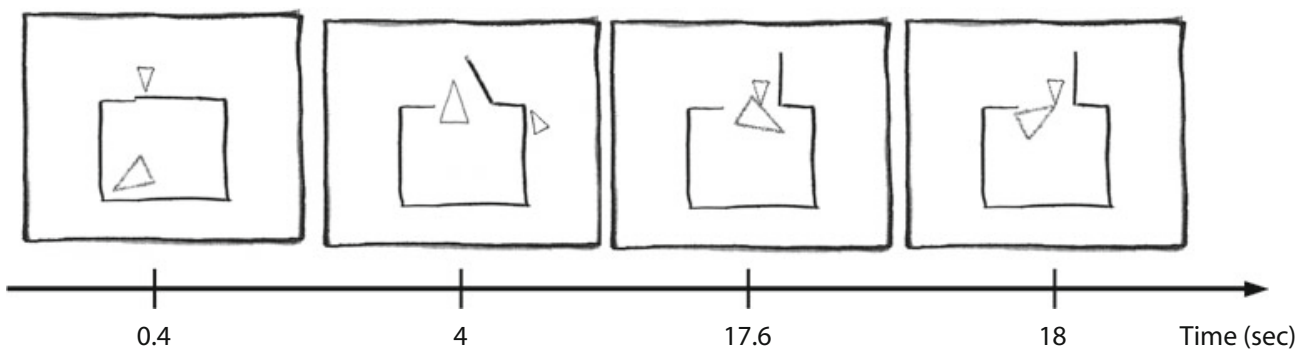

Figure 1. Still pictures of the theory-of-mind film "Surprising" for different time points. The small triangle knocks on a door and hides behind it while the big triangle is looking out of the house. After the big triangle has moved in again, the small triangle repeats its knocking and hiding behavior, slips in, and surprises the big triangle. 
Incongruent

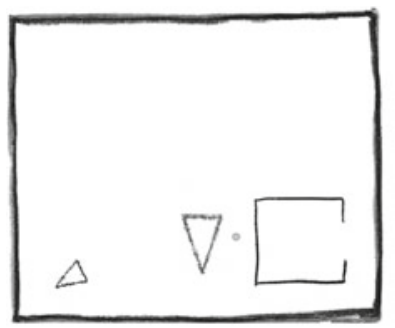

Figure 2. Examples of dot presentations in incongruent and congruent situations. In the picture shown on the left, the correct response would be right from the perspective of the participant but left from the perspective of the triangle. In the picture on the right side, the correct response would be right from both perspectives.

and right buttons of a gamepad. Head-to-monitor distance was approximately $60 \mathrm{~cm}$.

Nine film clips were taken from the Frith-Happé animations (Abell et al., 2000) and shortened to about $18 \mathrm{sec}$ while preserving the essential story line (Klein et al., 2009). All of these films, $20^{\circ}$ in width and $16^{\circ}$ in height, showed a red and blue triangle with heights of about $4^{\circ}$ and $2^{\circ}$ and widths of about $2^{\circ}$ and $1^{\circ}$, respectively.

During every film presentation, six time points were randomly selected, with the constraint that all the time points were separated by at least $1.5 \mathrm{sec}$ and that, in half of them, the red triangle was pointing upward and, in the other half, downward. At each time point, a filled gray circle with a diameter of $0.5^{\circ}$ appeared $2^{\circ}$ right or left of the center of mass of the red triangle for $30 \mathrm{msec}$. This constraint ensured that half of the dot presentations were in the same position relative to the red triangle when seen from the observer's and triangle's perspectives (congruent) and half were at different relative positions (incongruent). The short $30-\mathrm{msec}$ duration was chosen so that no triangle movement occurred during dot presentation. Figure 2 depicts the two congruency conditions. The side of dot appearance was determined pseudorandomly.

Film presentation was organized in blocks of films from the same category. Block order was balanced across participants. In each block, every film appeared 10 times in a pseudorandom sequence. This resulted in a total of 90 trials across all three blocks. Within participants, film category (R, GD, ToM) and congruency (incongruent, congruent) were varied.

Procedure. The participants were instructed to watch the films attentively in order to report on their content later. In addition, they were told that they should respond as quickly as possible to the relative location of the dot, as seen from their view. The participants were asked to press the right button if the dot appeared to the right of the triangle and the left button if it appeared on the left. Every trial started with a fixation cross $\left(1^{\circ}\right)$ at the center of the screen that was replaced by a film after $500 \mathrm{msec}$ of fixation. Importantly, all the films were presented without breaks, so that they differed from the films in the study by Klein et al. (2009) only in the dots that appeared.

In addition to the three experimental blocks, a training block with three other films was run. After each block, the participants reported what they had seen during the last presentations. These responses were required only as a means to ensure that the participants watched the films attentively, and were not further analyzed.

Data analysis. RT was measured from onset of the dot until a button was pressed. First, dot presentations with RTs larger than or equal to 1,500 msec were excluded (no responses). Next, wrong responses were excluded (wrong responses). Finally, all responses that differed in RT by more than two standard deviations from the mean for the participant were not analyzed (unfocused responses). RTs were subsequently averaged within participants for each film category and congruency condition separately. The differences in RTs between incongruent and congruent decisions (congruency effect) for each film category were then subjected to a repeated measures ANOVA with the factor of film category (R, GD, and ToM), which was followed up with paired $t$ tests.

\section{Results and Discussion}

The percentages of excluded trials were $8.49 \%, 4.96 \%$, and $3.59 \%$ for no, wrong, and unfocused responses. The congruency effect increased from the R to the GD to the ToM conditions (see Figure 3 and Table 1), which was statistically reflected in a main effect of film category $\left[F(2,46)=12.38, p<.01, \eta^{2}=.35\right]$. As can be seen from the $95 \%$ confidence intervals in Figure 3, only the GD and ToM conditions led to a significant congruency effect. Finally, paired Bonferroni-corrected $t$ tests revealed a significantly higher congruency effect in the ToM than in the GD condition $\left[t(23)=3.27, p_{2}<.01\right]$ and no difference between the GD and the R conditions $[t(23)=1.81$, $p_{2}>$.10]. The number of wrong responses was higher in the incongruent than in the congruent conditions and did not increase with shorter RTs, which is evidence against a speed-accuracy trade-off.

A comparison of the mean RTs for the GD and ToM conditions shown in Table 1 seems to suggest that the congruency effect was also caused by a decrease in RTs in the congruent condition and, therefore, that VSP adoption led not only to longer RTs in the incongruent conditions, but also to shorter RTs in the congruent conditions. Since, in the congruent ToM condition, the responses from the perspective of the participant and from the adopted perspective were the same, the faster of the two could have determined the RT. Post hoc, we calculated an ANOVA on the RTs for the incongruent and congruent conditions separately and found an effect of film condition for the incongruent conditions $\left[F(2,46)=4.18, p<.05, \eta^{2}=\right.$ $.15]$, but not for the congruent conditions $(F<1)$.

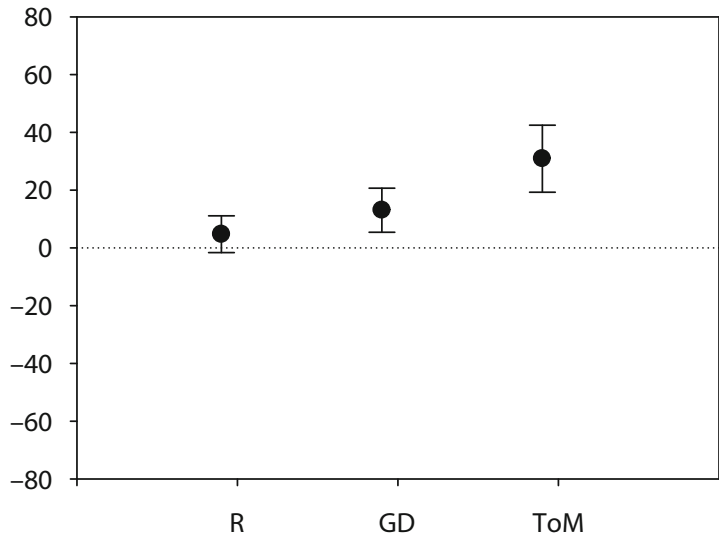

Film Category

Figure 3. Difference in mean reaction time (RT) between incongruent and congruent conditions as a function of film category. Whiskers indicate $\mathbf{9 5 \%}$ confidence intervals. R, random; GD, goal directed; ToM, theory of mind. 
Table 1

\begin{tabular}{|c|c|c|c|c|c|c|c|c|}
\hline \multicolumn{9}{|c|}{$\begin{array}{l}\text { Means and Standard Errors for Reaction T } \\
\text { and Wrong Response Rates (Number } \\
\text { Divided by the Number of Responses) } \\
\text { the Three Film Categories As a Fun }\end{array}$} \\
\hline \multirow[b]{3}{*}{ Film Category } & \multicolumn{4}{|c|}{ RT } & \multicolumn{4}{|c|}{ Wrong Response Rate } \\
\hline & \multicolumn{2}{|c|}{ Incongruent } & \multicolumn{2}{|c|}{ Congruent } & \multicolumn{2}{|c|}{ Incongruent } & \multicolumn{2}{|c|}{ Congruent } \\
\hline & $M$ & $S E$ & $M$ & $\overline{S E}$ & $M$ & $\overline{S E}$ & $M$ & $S E$ \\
\hline \multicolumn{9}{|c|}{ Experiment 1} \\
\hline Random & 523 & 15 & 518 & 15 & 3.54 & 0.76 & 2.33 & 0.47 \\
\hline Joal directed & 538 & 16 & 525 & 16 & 10.08 & 1.47 & 5.83 & 0.97 \\
\hline Theory of mind & 545 & 15 & 514 & 15 & 6.08 & 1.06 & 1.63 & 0.42 \\
\hline \multicolumn{9}{|c|}{ Experiment 2} \\
\hline andom & 317 & 8 & 318 & 8 & 3.21 & 0.55 & 3.08 & 0.62 \\
\hline Goal directed & 321 & 8 & 318 & 9 & 3.21 & 0.67 & 2.83 & 0.56 \\
\hline Theory of mind & 323 & 8 & 324 & 8 & 2.67 & 0.50 & 2.17 & 0.45 \\
\hline
\end{tabular}

In addition, interpreting this difference between the GD and ToM films for the congruent condition alone assumes that there is no general difference between RTs in the GD and ToM conditions. Making inferences in the GD condition, however, could be more difficult than mentalizing in the ToM conditions, and this would lead to generally elevated RTs in the GD condition. The higher error rates in the GD condition support this interpretation. This difference in RT between film categories is controlled for when the difference between incongruent and congruent conditions within each film condition is used.

This pattern of results suggests that people spontaneously adopt the perspective of an agent and code responses relative to this perspective. This VSP taking was increased for films with mentalizing content (see the General Discussion section). However, the present experiment did not exclude the possibility that these response differences were caused by superficial visual differences in the animations. To exclude this alternative interpretation, Experiment 2 was performed.

\section{EXPERIMENT 2}

In Experiment 2, we wanted to rule out the possibility that superficial visual differences between the animations - for example, different positions of the triangle - caused the differences in RTs in Experiment 1. Therefore, 25 different participants saw the same triangle-dot situations from Experiment 1; that is, each participant in Experiment 2 was matched to 1 participant in Experiment 1. Crucially, because only still pictures were shown, agency attribution and mentalizing should not occur. Therefore, if a congruency effect still occurred, it would suggest that the effect in Experiment 1 was caused by differences in visual difficulty. However, observing no congruency effect would support the interpretation of Experiment 1 that it was, indeed, processes associated with the attribution of agency or mental states that caused the congruency effect.

\footnotetext{
Method

Participants, Apparatus, Stimuli, Design, Procedure, and Analysis. Twenty-five participants $(17$ females, mean age $=$
}

30 years) took part in Experiment 2. The number of participants was increased in order to replace 1 participant who had more than $80 \%$ wrong responses. All other conditions were as reported in Experiment 1 , except that no eyetracking was used and, in each trial, only six still pictures (the same decision situations as in Experiment 1) were shown. Each presentation started with a display of the situation without the dot. After a random interval of $500-1,000 \mathrm{msec}$, the dot was added, and it disappeared, as in Experiment 1, after $30 \mathrm{msec}$. The participants responded as quickly as possible with the "s" key if the dot occurred left of the triangle and with the "l" key if the dot occurred to the right.

\section{Results and Discussion}

Exclusion rates were $0.76 \%, 2.93 \%$, and $3.74 \%$ this time. RTs were considerably lower than in Experiment 1, which was probably caused by the additional task of understanding the story and the higher temporal uncertainty of the dot in Experiment 1. Importantly, RTs were nearly identical in every condition (see Table 1 ). This was corroborated by a nonsignificant ANOVA $[F(2,46)=1.90$, $p>.10]$ and a significant difference in effect size between the two experiments $(Z=2.88, p<.01) .{ }^{1}$ Therefore, when no story was provided, the same triangle-dot situations as those in Experiment 1 did not lead to a difference between the film conditions. Also, the number of wrong responses differed only slightly between the conditions.

One could argue that the generally shorter RTs in Experiment 2 did not allow for any perspective effect to take place. This seems rather unlikely, given that the triangles, without the dot, were always presented for at least $500 \mathrm{msec}$ and this should have been enough time for VSP adoption to take place. However, Experiment 2 rules out the alternative interpretation that the effect found in Experiment 1 was caused by low-level visual differences.

\section{GENERAL DISCUSSION}

In Experiment 1, the participants were presented with films that did or did not invoke agency attribution to a geometric figure. During films in which agency attribution occurred, the participants were expected to adopt the VSP of the agent. Adoption of the triangle's perspective should lead to interference when the red triangle pointed downward and the triangle's and participant's perspectives 
differed, but not when the triangle pointed upward. This conflict would require time to inhibit the inappropriate response of the adopted perspective. Similarly, when attribution of agency occurred in congruent conditions, the activation of two congruent responses led to shorter RTs. The largest congruency effect was found in the ToM condition. Experiment 2 ruled out the possibility that the findings in Experiment 1 were caused by some superficial differences between visual properties among the three categories of films.

One interpretation of this could be that mental state attribution adds to the effect of agency attribution and, therefore, leads to stronger VSP taking. An alternative interpretation is that the strength of agency attribution increased in the ToM condition, as compared with the GD condition. According to this interpretation VSP taking is associated with agency attribution alone. The present experiments did not rule out this possibility. However, the former interpretation seems more likely, because differences between ToM and GD films have typically been described in terms of mentalizing, but not agency (e.g., Abell et al., 2000). Furthermore, even though a significant effect of VSP taking was found for the GD films, but not for the R films, VSP taking was not significantly larger during GD films than during R films. In contrast, VSP taking was significantly larger during ToM films, which supports the importance of mentalizing for VSP taking.

Response slowing in the incongruent GD and ToM conditions was not caused by disposing of the perspective of the participant wholly, because, in this case, no conflict would occur. In contrast, these results reflect the coactivation of two VSPs, the adopted perspective and the perspective of the participant.

The fact that VSP taking occurred also during triangle animations shows that VSP taking is not dependent on the presence of a human body, as in Thomas et al. (2006), where no movements were shown. In the presence of agency cues, VSP taking in the present study occurred spontaneously, as in Thomas et al. (2006) and Tversky and Hard (2009), even if it was not required by the task. The spontaneous involvement of VSP processes in the ToM condition suggests that mental perspective taking not only involves abstract reasoning about the other's state, but also entails adopting the actual VSP. It will be a question for further research to determine the minimal requirements for stimuli contents to evoke VSP taking and whether the present films are a representative sample of those contents.

\section{AUTHOR NOTE}

This work was supported by the Deutsche Forschungsgemeinschaft (DFG) excellence initiative "CoTeSys." I thank Uta Frith and Francesca Happé for making these animations available to me, and Ulrike Hahn, Uta Frith, and two anonymous reviewers for helpful suggestions on an earlier version of the manuscript. I am also in debt to Jared Pool and Katrin Rebekka Scharpf for helpful comments on the topic. Correspondence concerning this article should be addressed to J. Zwickel, Department of Psychology, Ludwig-Maximilians-Universität München, Leopoldstrasse 13, 80802 Munich, Germany (e-mail: zwickel@psy.uni -muenchen.de).

\section{REFERENCES}

Abell, F., Happé, F., \& Frith, U. (2000). Do triangles play tricks? Attribution of mental states to animated shapes in normal and abnormal development. Cognitive Development, 15, 1-16. doi:10.1016/S0885 -2014(00)00014-9

Aichhorn, M., Perner, J., Kronbichler, M., Staffen, W., \& LaDURNER, G. (2006). Do visual perspective tasks need theory of mind? NeuroImage, 30, 1059-1068.

APPERLY, I. A. (2008). Beyond simulation-theory and theory-theory: Why social cognitive neuroscience should use its own concepts to study "theory of mind." Cognition, 107, 266-283. doi:10.1016/j .cognition.2007.07.019

Castelli, F., Happé, F., Frith, U., \& Frith, C. (2000). Movement and mind: A functional imaging study of perception and interpretation of complex intentional movement patterns. NeuroImage, 12, 314-325. doi:10.1006/nimg.2000.0612

Frith, C. D., \& Frith, U. (2006). How we predict what other people are going to do. Brain Research, 1079, 36-46. doi:10.1016/j .brainres.2005.12.126

Jackson, P. L., Meltzoff, A. N., \& Decety, J. (2005). How do we perceive the pain of others? A window into the neural processes involved in empathy. NeuroImage, 24, 771-779. doi:10.1016/j .neuroimage.2004.09.006

Johnson, S. C. (2003). Detecting agents. Philosophical Transactions of the Royal Society B, 358, 549-559. doi:10.1098/rstb.2002.1237

Klein, A., Zwickel, J., Prinz, W., \& Frith, U. (2009). Animated triangles: An eye tracking investigation. Quarterly Journal of Experimental Psychology, 62, 1189-1197. doi:10.1080/17470210802384214

Rosenthal, R. (1997). Meta-analytic procedures for social research. London: Sage.

Thomas, R., Press, C., \& Haggard, P. (2006). Shared representations in body perception. Acta Psychologica, 121, 317-330. doi:10.1016/j .actpsy.2005.08.002

Tversky, B., \& Hard, B. M. (2009). Embodied and disembodied cognition: Spatial perspective-taking. Cognition, 110, 124-129. doi:10.1016/j.cognition.2008.10.008

\section{NOTE}

1. The calculation was done as suggested in Rosenthal (1997).

(Manuscript received December 7, 2008; revision accepted for publication July 13, 2009.) 